General Conference (Part A)

\title{
DYNAMIC RESOURCE ALLOCATION FOR LEO SATELLITE SYSTEMS WITH QOS PROVISION FOR AN IPv6 NETWORK
}

\author{
E. Del Re IEEE Senior Member, R. Fantacci IEEE Senior Member, \\ T. Pecorella IEEE Student Member, L.S. Ronga IEEE Member, F. Castellani \\ e-mail: \{delre,fantacci,pecos,ronga,franz\}@lenst.die.unifi.it \\ Università degli Studi di Firenze, E.E. Dept. - LENST Lab. \\ Via di Santa Marta 3, 50139 FIRENZE, ITALY - Ph. \& Fax +39-055-4796485
}

\begin{abstract}
The approach presented in this paper aims to obtain a QoS for IPv6 protocol over LEO satellites. Most of the switching and access operations in the proposed system will require the intelligent functions to be located on earth.

We can allocate links at different bit-rates and no particular assumptions have been made as concerns the access protocol in order to maintain the presented work at a general level. Both Controlled Load and Not-Controlled Load traffic have been considered.

The behavior of the proposed system shows that the utilization and the delays are strictly related. The simulations show that large delays correspond to small values of utilization while short delays have the drawbacks of bandwidth waste. Results point out that, as concerns the privileged traffic classes of NCL are subject to delays very close to those obtained by the CL traffic while maintaining high link utilizations.
\end{abstract}

\section{Introduction}

In communication systems of the third generation an high degree of integration is envisaged. Terminals will be able to access the network from several different environments using heterogeneous sub-networks. For the provision of high data-rate services terrestrial networks are to be preferred, however, in some circumstances, satellite access is the most convenient solution, in particular LEO orbits offer a global coverage and low delays in comparison to other satellite systems.

Studies concerning the characteristics of traffic in global digital networks foresee that IP protocol will be largely used for the delivery of services to the users, while ATM will play a major role in the backbones. The new generation of IP, IPv6 [1], provides some interesting properties concerning integration on mobile networks and classification of flows. The development of this protocol suite is foreseen for the delivery of the great variety of services provided by future communication systems.

The provision of QoS is bandwidth consuming and the bandwidth is a valuable resource in satellite systems. In our work we try to find a compromise between an efficient utilization of the links and an acceptable QoS control.

At present the support for the quality of service in the Internet is not fully implemented [2][3]. The standardization process of some methods for QoS support over IP terrestrial networks is not completed.

The approach presented in this paper aims to obtain a QoS for IPv6 protocol over LEO satellites. Most of the switching and access operations in the proposed system will require the intelligent functions to be located on earth, in agreement with the extremely stringent power and weight constraints imposed to LEO satellites.

In order to achieve the needed QoS, we can rely on the integrated or differentiated services [4].

The source of traffic considered in the model is of the aggregated type. Single user traffic will be considered in a future development of the proposed system. The aggregated traffic derives from a number of sources of different kind: multimedia services and TCP applications like file transfer, interactive terminals and WEB browsing.

As concerns the traffic management, some flows explicitly require QoS provision which is obtained in our system using integrated services; we implemented only the CL (Controlled Load) [5] service for this purpose. In our opinion the majority of future real-time applications will be adaptive and in the modeled satellite system the implementation of the guaranteed service [6] would lead to unacceptable wastes of bandwidth.

The traffic that does not use the CL service is called hereafter NCL (Not Controlled Load).

In the $\mathrm{CL}$ traffic the waste of bandwidth cannot be 


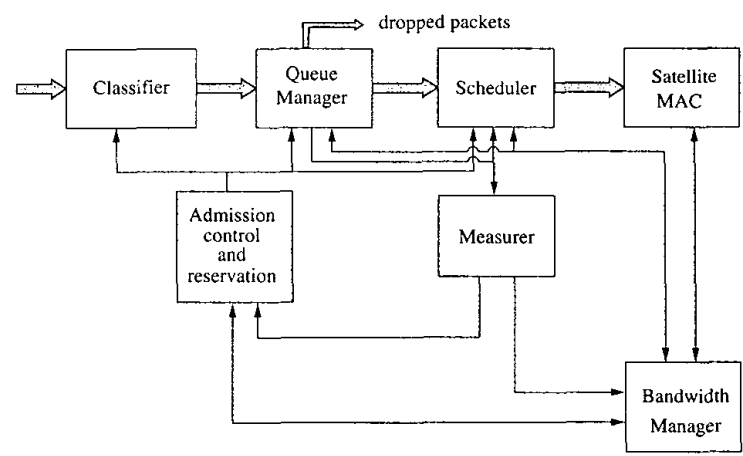

Figure 1: The block diagram of our system

avoided, so we decided not to allow the NCL traffic to waste bandwidth. This choice leads to greater delays for the NCL packets.

In order to provide some QoS in the NCL traffic, we implemented the differentiated policy in this class of traffic, so we can provide a QoS support also for the NCL traffic.

The channel resources are modeled in a way similar to the hierarchical structure proposed by the ETSI $\alpha$ group. We can allocate links at different bit-rates and no particular assumptions have been made as concerns the access protocol in order to maintain the presented work at a general level.

Our objective can be summarize as follow:

1. provide support for QoS;

2. maximize the utilization of the allocated links;

3. minimize the total number of the allocated links in order to grant access to other users to the satellite and to minimize channel interference;

4. deny waste of bandwidth for the NCL traffic.

\section{The system}

In the fig. 1 is shown the scheme of our system. The six blocks are described in the following:

Classifier: it execute the classification of the packets and selects the service to be received. The classification is based on IPv6 flow labels for CL traffic and DS. field for the NCL one.

Queue manager: its duty is to check the queue state and drop or mark packets when necessary. This block was not fully implemented in our simulations.
Scheduler: it picks out from the queue the packet to be sent. We used a modified version of the H-FSC algorithm [7]. The H-FSC algorithm, as well as others scheduling algorithms can not be successfully applied in this case for three main reasons: the bit-rate of the channel is variable, the total bit-rate is composed by a number of links and, finally, the characterization of the NCL traffic does not fit the requirements of the H-FSC algorithm.

The choice of the H-FSC is due by a more flexible resource management than H-PFQ algorithms allows by decoupling the delay and bandwidth allocation.

To process the CL traffic, we modified the H-FSC algorithm as follows:

- the re-ordering of the packets is avoided;

- the higher bit-rate links are preferred.

To process the NCL traffic only the link-sharing criteria is used.

Measurer: it measures the number of bits arrived to the node in a time slot $T_{S}$. This measure is needed either by the admission control algorithm and as an estimate of the CL bit-rate for the bandwidth manager. We adopted two measurement mechanisms: the time-window [8] and the exponential averaging.

Admission control and reservation: the admission control algorithm adopted is the measured sum [8].

The choice is due to the easiness its implementation and to its operational costs together to its good performances

This algorithm admits a new flow when the following condition happens:

$$
S+r<0.9 \cdot B_{\mathrm{CL}}
$$

where $r$ is the declared mean bit-rate of the new flow, $B_{\mathrm{CL}}$ is the total allocated bit-rate and

$$
S=\min \left\{R_{s}, R_{\mathrm{CL}}\right\},
$$

where $R_{s}$ is the measured load of the existing CL traffic and $R_{\mathrm{CL}}$ is the sum of the bit-rates announced by the admitted flows.

When the condition in (1) is not satisfied, the request for a new link is sent to the Bandwidth Manager.

Bandwidth manager: it talks directly to the satellite MAC. Its task is to decide weather to allocate new satellite links and when to release old ones. A decision is 
taken every $T_{a}$ seconds. To improve the performances of the system $T_{a}$ should be as small as possible, nevertheless small values of $T_{a}$ are lower-bounded by the $T_{\max }$ delay (see later).

We make the assumption that the requests of bandwidth to the MAC layer has to be limited. The limitation is imposed on the total available bit-rate and on the number of links for a given bit-rate. The first one leads to a probability of allocation failure increasing with the total amount of allocated bandwidth. The second limitation tends to reduce the total number of allocated links by preferring a small number of high bit-rate links to a large number of low bit-rate links.

The granularity of the allocated links is not constant but is adapted to the total allocated bit-rate. The proposed allocation strategy chooses small steps of bit-rate when the total requested bandwidth is small, and larger steps when the total requested bandwidth is high.

Every $T_{a}$ seconds we get

$$
\text { rate }=\frac{Q}{T_{a}}+\text { Rnew }_{\mathrm{CL}},
$$

where $Q$ is the number of bits waiting in queue and Rnew $w_{\mathrm{CL}}$ is derived from flows blocked by the admission control test as explained in (1).

As a matter of facts, $Q / T_{a}$ is the bit-rate needed to send all the packets in queue in $T_{a}$ seconds.

The variable rate is compared with $W$, where $W$ is derived from:

$$
W=\min \left\{B_{\mathrm{CL}}, R_{\mathrm{CL}}, R_{s} \rho\right\},
$$

where $\rho(\rho>1)$ is necessary to reserve some more bandwidth for the CL traffic. The quantity ReqRate defined by

$$
\text { ReqRate }=\min \{\text { rate }, W\},
$$

represents the effective rate to be requested to MAC layer. The quantity ReqRate, compared with the total allocated bit-rate $B_{\text {tot }}$ is used to decide whether to release or request links.

Note that with this method the needed bandwidth is predicted by considering the CL traffic only, while for the NCL traffic we measure the queued packets; this allows not to have bandwidth waste for the NCL traffic.

\section{The simulated LEO system}

As concerns the MAC layer on the LEO satellite, the following model is adopted. The probability of rejection of a re- quest is related both to the requested bit-rate and the present load of the system.

The request will be acknowledged with a uniform random delay between $T_{\min }$ and $T_{\max }$ which take into account the delays due to propagation in the satellite channel, latency for the request processing and eventual collisions on the request channel.

We put

$$
\left\{\begin{array}{l}
T_{\min }=19 \mathrm{~ms} \\
T_{\max }=42 \mathrm{~ms}
\end{array}\right.
$$

Given these values, we have chosen the value for $T_{a}$ to be $50 \mathrm{~ms}$.

The satellite channel was modeled in a way similar to the ETSI $\alpha$ group: eight different link type, from 32 to $256 \mathrm{kbps}$ arranged in a tree structure, in a way that higher bit rate channels can be splitted into two lower bandwidth links. No assumptions have been made on the access method.

We divided the NCL traffic in 8 service classes, each one characterized by a different delay.

Recent studies $[9,10,11]$ have shown that for the network traffic a self-similar model is more realistic than the traditional Poisson model. This model involves that bursts can be distributed over a large scale. Self-similar traffic can be obtained by the superposition of many strictly alternating i.i.d. ON/OFF sources. Each source is heavy-tailed distributed, following the Pareto distribution described by two parameters: the location $x_{0}$ and the shape $\alpha$. These parameters are connected with the mean $\mu$ by the following relationship:

$$
\mu=\frac{\alpha}{\alpha-1} x_{0} .
$$

To generate the NCL traffic at various bit-rate we consider the sum of several sources of this type.

CL traffic aggregation also can be modeled as self-similar; we considered each flow as an ON/OFF source. In tab. 1 are shown the models used in our simulations (see [12] and ETSI recommendations). The Poisson model has been also used for comparisons.

We keep a queue for each flow (CL traffic) and traffic class (NCL traffic). The maximum length of the queue associated with a flow is determined by the parameters announced by the flow itself. The maximum length of the queue associated with a traffic class is determined by the bit-rate allocated for the whole NCL traffic. We choose to drop packets when the queue is full.

\section{Simulation results}

In the simulations the utilization of the satellite links Vs the packet delay is investigated. 


\begin{tabular}{|c|c|c|c|c|}
\hline Distribution & $\mu_{\mathrm{ON}}(\mathrm{ms})$ & $\mu_{\mathrm{ON}}(\mathrm{ms})$ & Peak rate & $\alpha$ \\
\hline \hline Pareto & 400 & 400 & $76 \mathrm{kbps}$ & 1.5 \\
\hline Pareto & 40 & 360 & $304 \mathrm{kbps}$ & 1.1 \\
\hline Pareto & 313 & 2925 & $76 \mathrm{kbps}$ & 1.1 \\
\hline Exponential & 313 & 325 & $76 \mathrm{kbps}$ & \\
\hline Exponential & 20 & 180 & $304 \mathrm{kbps}$ & \\
\hline Exponential & 3000 & 3000 & $64 \mathrm{kbps}$ & \\
\hline
\end{tabular}

Table 1: ON/OFF sources model used in our simulations for CL traffic.

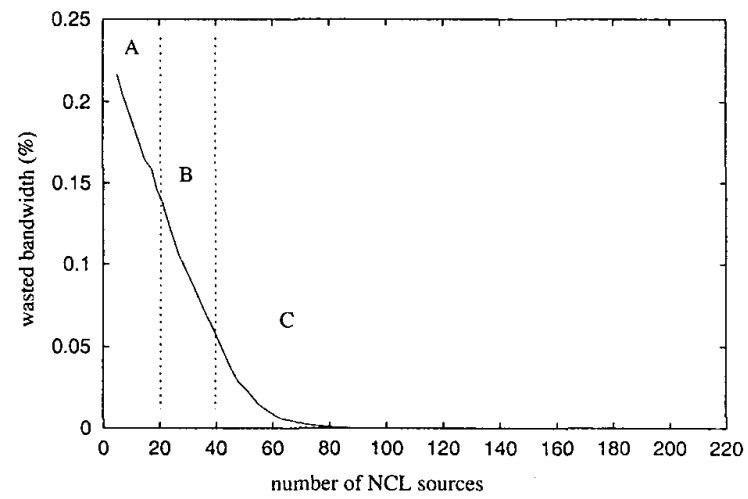

Figure 2: The utilization of the allocated links.

In the simulations we considered a fixed number of CL sources and we plot the performances of the proposed system with a variable number of NCL traffic sources, i.e. a variable NCL traffic rate. The performance indexes are the amount of wasted satellite bandwidth (i.e. the allocated but unused fraction of bandwidth) and the CL traffic queue and delivery delay. A typical behavior of the the system is plotted in figs. 2 and 3.

The mean delay has a peak; this can be attributed to our choice to avoid a re-ordering of the CL packets in an environment characterized by several links. This peak can be eliminated, but we do not have anymore a monotonous curve for the utilization.

In the presence of predominant $\mathrm{CL}$ traffic the delay can be maintained low only at expense of a low utilization (region A of the figures).

As the NCL traffic increases (region B) the delay gradually increases but the waste lowers. This behavior can be explained by the fact that the NCL traffic covers the holes not filled by CL traffic.

After a threshold the heavy load of NCL traffic impose a high bandwidth allocation; this CL traffic takes advantage from that since it has a higher priority. In this region (region

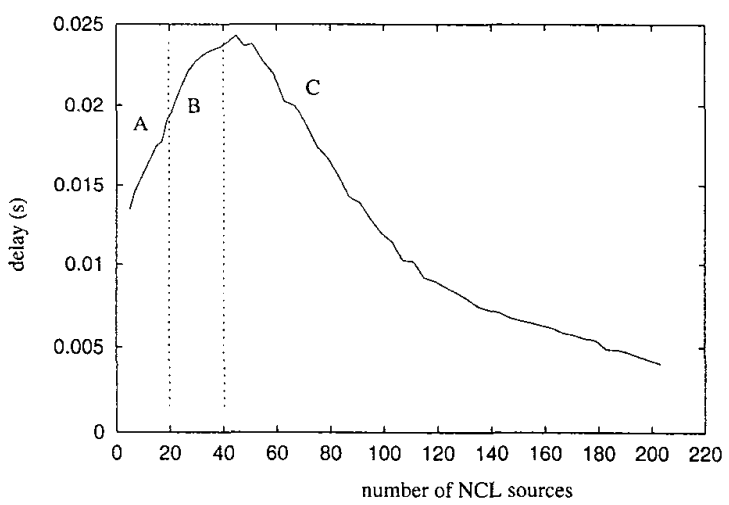

Figure 3: The mean delay of the CL packets.

C) the CL flows always find a way to be quickly delivered. The utilization in this region is very high due to lack of bandwidth holes while the CL delay decreases.

It is important to highlight that, as concerns NCL traffic, the mean delays are maintained sufficiently small, and the privileged traffic classes are subject to delays very close to those obtained by the CL traffic.

\section{Conclusions}

We presented a system able to manage both $\mathrm{CL}$ and NCL IPv6 traffic flows over LEO satellite links where a shared medium is employed at physical level. The bandwidth allocation is dynamic and a QoS support is provided.

The results show that it is possible get low delays and high utilization in the presence of prevalent NCL load, while when the majority of the traffic is $\mathrm{CL}$, small delays correspond to a poor utilization.

Several others scheduling models will be considered in the near future.

The system studied in this work will be also applied to single (not aggregated) CL traffic sources, and the performances will be published shortly.

Another interesting extension is under development for NCL traffic type only, implementing the Differentiated Services model and Advanced Flow Control.

\section{References}

[1] S. Deering and R. Hinden, "Internet Protocol, Version 6 (IPv6) Specification," RFC 2460, Internet Engineering Task Force, Dec. 1998. 
[2] "Integrated services (intserv)," http://www.ietf.org/html.charters/intserv-charter.html.

[3] "Differentiated services (diffserv)," http://www.ietf.org/html.charters/diffservcharter.html.

[4] S. Blake, D. Black, M. Carlson, E. Davies, Z. Wang, and W. Weiss, "An Architecture for Differentiated Services," RFC 2475, Internet Engineering Task Force, Dec. 1998.

[5] J. Wroclawski, "Specification of the Controlled-Load Network Element Service," RFC 2211, Internet Engineering Task Force, Sept. 1997.

[6] S. Shenker, C. Partridge, and R. Guerin, "Specification of Guaranteed Quality of Service," RFC 2212, Internet Engineering Task Force, Sept. 1997.

[7] I. Stoica, H. Zhang, and T.S.E. Ng, "A Hierarchical Fair Service Algorithm for Link-Sharing, Real-Time and Priority Services," in Proceedings of ACM SIGCOMM '97, Aug. 1997.

[8] S. Jamin, S.J. Shenker, and P.B. Danzig, "Comparison of Mearurement-based Admission Control Algorithms for Controlled-Load Service," in Proceedings of INFOCOM '97, Apr. 1997.

[9] M.E. Crovella and A. Bestavros, "Self-Similarity in World Wide Web Traffic: Evidence and Possible Causes," IEEE/ACM Transactions on Networking, vol. 5, no. 6, pp. 835 845, Dec. 1997.

[10] V. Paxson and S. Floyd, "Wide Area Traffic: The Failure of Poisson Modeling," IEEE/ACM Transactions on Networking, vol. 3, no. 3, pp. 226-240, June 1995.

[11] W. Willinger, M.S. Taqqu, R. Sherman, and D.V. Wilson, "Self-Similarity Through High-Variability: Statistical Analysis of Ethernet LAN Traffic at the Source Level," IEEE/ACM Transactions on Networking, vol. 5, no. 1, pp. 71-86, Feb. 1997.

[12] C. Jin and S. Jamin, "Design, Implementation, and End-to-End Evaluation of a Measurement-Based Admission Control Algorithm for Controlled-Load Service," in Proceedings of NOSSDAV '98, July 1998. 